\title{
Coordinated Precoding for D2D Communications Underlay Uplink MIMO Cellular Networks
}

\author{
Bing Fang, Zuping Qian, Wei Zhong, Wei Shao, and Hong Xue \\ College of Communications Engineering, PLA University of Science and Technology, Nanjing 210007, China \\ Correspondence should be addressed to Bing Fang; bingfang_ch@163.com
}

Received 8 November 2015; Accepted 13 March 2016

Academic Editor: Lin Gao

Copyright (c) 2016 Bing Fang et al. This is an open access article distributed under the Creative Commons Attribution License, which permits unrestricted use, distribution, and reproduction in any medium, provided the original work is properly cited.

\begin{abstract}
We study the coordinated precoding problem for device-to-device (D2D) communications underlay multiple-input multipleoutput (MIMO) cellular networks. The system model considered here constitutes multiple D2D user pairs attempting to share the uplink radio resources of a cellular network. We first formulate the coordinated precoding problem for the D2D user pairs as a sum-rate maximization (SRM) problem, which is subject to a total interference power constraint imposed to protect the base station (BS) and individual transmit power budgets available for each D2D user pair. Since the formulated SRM problem is nonconvex in general, we reformulate it as a difference convex- (DC-) type programming problem, which can be iteratively solved by employing the famous successive convex approximation (SCA) method. Moreover, a proximal-point-based regularization approach is also pursued here to ensure the convergence of the proposed algorithm. Interestingly, the centralized precoding algorithm can also lend itself to a distributed implementation. By introducing a price-based interference management mechanism, we reformulate the coordinated precoding problem as a Stackelberg game. Then, a distributed precoding algorithm is developed based on the concept of Stackelberg equilibrium (SE). Finally, numerical simulations are also provided to demonstrate the proposed algorithms. Results show that our algorithms can converge fast to a satisfactory solution with guaranteed convergence.
\end{abstract}

\section{Introduction}

Recently, device-to-device (D2D) communications underlay cellular networks have received much attention due to their potential power to provide higher data rates and larger system capacity to meet the overwhelming demands of "Big Data" age $[1,2]$. D2D communication in cellular networks is defined as direct communication between two mobile users and is likely to be a promising paradigm for the next generation cellular technologies (i.e., 5G). Introducing D2D communication in cellular networks will dramatically improve spectrum utilization, network throughput, and energy efficiency, while facilitating new peer-to-peer and location-based social networking applications at the same time. However, enabling the D2D communication mode in the traditional cellular networks will also bring many challenges to the long-standing cellular architectures; the main ones among them are radio resource allocation and interference management [3-5].

Through taking the advantage offered by multiple-input multiple-output (MIMO) technologies, the D2D user pairs and the regular cellular users can cooperate in the same timefrequency resource block, while keeping the interference between them to a tolerable level [5-8]. For example, the authors in [5] considered the resource allocation problem for D2D communications underlay MIMO cellular networks. With a goal to maximize the achievable sum-rate, they exploit the MIMO transmission advantages, that is, through joint beamforming and power control, to allocate the available radio resources and control the interference at the same time. Paper [8] utilizes the spatial freedom offered by MIMO to avoid interference from cellular downlink transmission to the underlay D2D users, with the aim of guaranteeing the D2D communication performance. As the works done in $[9,10]$, this issue has also been studied from a game-theoretic perspective for a distributed implementation. However, the coordinated precoding problem to allocate the available cellular radio resource to the D2D user pairs for the general case, that is, all nodes considered in the system employing multiple antennas, is of huge nonconvex complexity and deemed as extremely challenging, especially when the social 
performance criteria, such as the achievable sum-rate and the total mean square error (MSE), are considered.

A common model for studying the coordinated precoding problem for $\mathrm{D} 2 \mathrm{D}$ communications underlaying $\mathrm{MIMO}$ cellular networks is the MIMO interference channel (IFC), where multiple transmitters simultaneously communicate with their respective receivers over a common frequency band [11-15]. Coordinated precoding for the MIMO IFCs has been recognized as a promising approach to improving the system performance. According to the level of cooperation, the coordinated precoding problems can be roughly classified into two categories, that is, MIMO cooperation and interference coordination. In the MIMO cooperation case, the transmitters cooperate in data transmission by sharing all the channel state information (CSI) and data signals. However, in the interference coordination case, the transmitters only need to coordinate in the transmission strategies for mitigating the interchannel interference. Compared with MIMO cooperation, interference coordination scheme requires the CSIs to be shared only and hence induces less information exchanges among the transmitters.

In this paper, we keep a focus on the coordinated precoding problem for D2D communications underlay uplink MIMO cellular networks, where an interference coordination scheme is adopted. The system model considered here consists of multiple D2D user pairs attempting to share the uplink radio resources of a cellular network, where all modes are assumed to be equipped with multiple antennas. The coordinated precoding problem for such a system is formulated as a sum-rate maximization (SRM) problem subject to individual transmit power constraints. At the same time, since the base station (BS) has the power to control all D2D user pairs, thus a maximum tolerable interference power constraint is further imposed to protect the signal received by itself. Hence, the SRM problem for the proposed system is subject to both individual transmit power constraints and a coupling interference power constraint.

Since the formulated SRM problem is nonconvex in general, we equivalently reformulate it as a difference convex(DC-) type programming problem, which can be iteratively solved by employing a successive convex approximation (SCA) method $[16,17]$. With the SCA method, the nonconvex part of the SRM problem is locally linearized to its firstorder Taylor expansion. Thus, relying on solving a series of convexified optimization problems, a centralized iterative precoding algorithm is developed. In addition, a proximalpoint-based regularization is also pursued in this work to ensure the convergence of the proposed algorithm without requiring any special restrictions on antenna configurations and the channel ranks. Interestingly, the centralized iterative precoding algorithm is naturally suitable for a distributed implementation, because the convexified problem has a separable objective function. Then, by introducing a pricebased interference management mechanism to overcome the coupling interference power constraint, we reformulate the coordinated precoding problem as a Stackelberg game [18-21]. Then, a distributed precoding algorithm is further developed based on the concept of Stackelberg equilibrium (SE). Numerical simulations are also provided to demonstrate the proposed algorithms. Results show that our algorithms can converge fast to a satisfactory solution with guaranteed convergence. In summary, main contributions of the present paper can be listed as follows:

(i) A coordinated precoding framework is established for the D2D communications underlay uplink MIMO cellular networks. Moreover, the coordinated precoding problem is formulated as a SRM problem, about which the compute complexity is analyzed.

(ii) Based on the SCA method, a centralized iterative precoding algorithm is developed relying on solving a series of convexified optimization problems. In addition, a proximal-point-based regularized approach is also pursued to ensure the convergence of the proposed algorithm without requiring any special restrictions on antenna configurations and the channel ranks.

(iii) By the aid of the price-based interference management mechanism to overcome the coupling interference power constraint, we reformulate the coordinated precoding problem as a Stackelberg game. Then, a distributed precoding algorithm is further developed based on the concept of SE.

(iv) Numerical simulations are also provided to demonstrate the proposed algorithms. Results show that the proposed algorithms can converge fast to a satisfactory solution with guaranteed convergence.

The rest of this paper is organized as follows: in Section 2, we introduce the system model and formulate the SRM problem; in Section 3, a centralized iterative precoding algorithm is developed; and a distributed precoding algorithm is also designed in Section 4; in Section 5, we evaluate the proposed algorithms via computer simulations; the present paper is concluded in Section 6.

Notations. Bold uppercase letters denote matrices and bold lowercase letters denote vectors; $\mathbb{C}^{m \times n}$ defines the space of all $m \times n$ complex matrices; $\mathbf{A} \succeq 0$ means that matrix $\mathbf{A}$ is positive semidefinite; Hermitian transpose of matrix $\mathbf{A}$ is represented as $\mathbf{A}^{H} ;|\mathbf{A}|,\|\mathbf{A}\|_{F}$, and $\operatorname{Tr}(\mathbf{A})$ mean the determinant, the Frobenius norm, and the trace of matrix $\mathbf{A}$, respectively; and $\log (\cdot)$ denotes the natural logarithm.

\section{System Model and Problem Statement}

In this section, we first provide the system model and then formulate the coordinated precoding problem as a SRM problem, about which the compute complexity is analyzed.

2.1. System Model. In this work, we study the coordinated precoding problem for the D2D communications underlay uplink MIMO cellular networks. As shown in Figure 1, the system model considered here consists of $k$ D2D user pairs attempting to share the uplink radio resources of a cellular BS. For simplicity, we assume that only one BS coexists with the D2D user pairs. However, this model can be easily extended 


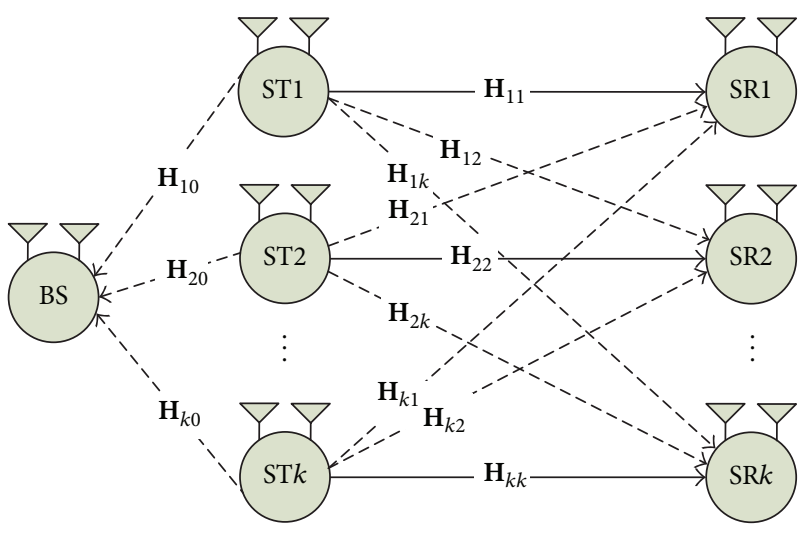

FIGURE 1: System model of $k$ D2D user pairs underlaying uplink MIMO cellular networks.

to the scenarios where multiple BSs are present. We also assume each D2D user pair $i$ is comprised of a secondary transmitter $(\mathrm{ST} i)$ and an intended secondary receiver (SR $i)$, where $i \in \mathscr{K}$ and the set of all D2D user pairs is defined as $\mathscr{K}=\{1,2, \ldots, k\}$. As mentioned before, a general case where all nodes in the proposed system are equipped with multiple antennas is considered. Specifically, it is assumed that the BS is equipped with $M_{0}$ antennas, and D2D user pair $i$ is equipped with $M_{i}$ antennas at the receiver and $N_{i}$ antennas at the transmitter. In addition, a quasi-static frequency-flat fading environment for all communication links is assumed.

We further assume that $\mathbf{x}_{j} \in \mathbb{C}^{N_{j}}, \forall j \in \mathscr{K}$, is the complex transmit signal of ST $j$. Then, the signal received at SR $i$ can be expressed as

$$
\mathbf{y}_{i}=\sum_{j=1}^{k} \mathbf{H}_{j i} \mathbf{x}_{j}+\mathbf{n}_{i}, \quad \forall i \in \mathscr{K}
$$

where $\mathbf{y}_{i} \in \mathbb{C}^{M_{i}}$ denotes the received signal at SRi, $\mathbf{n}_{i} \in$ $\mathbb{C}^{M_{i}}$ denotes the received additive noise plus the intercell interference from regular cellular users at SRi, and $\mathbf{H}_{j i} \in$ $\mathbb{C}^{M_{i} \times N_{j}}$ denotes the complex channel matrix from ST $j$ to SR $i$. Then, the achievable data rate of the $i$ th $\mathrm{D} 2 \mathrm{D}$ user pair can be given as follows:

$$
R_{i}(\mathbf{Q})=\log \left|\mathbf{H}_{i i} \mathbf{Q}_{i} \mathbf{H}_{i i}^{H}+\mathbf{Z}_{i}\right|-\log \left|\mathbf{Z}_{i}\right|, \quad \forall i \in \mathscr{K},
$$

where $\mathbf{Q}$ denotes the strategy profile of all $\mathrm{D} 2 \mathrm{D}$ user pairs and is defined as $\mathbf{Q}=\left\{\mathbf{Q}_{i}\right\}_{i=1}^{k}$ and $\mathbf{Z}_{i}$ is the covariance matrix of the additive noise plus all interference at $\mathrm{SR} i$, which is denoted as

$$
\mathbf{Z}_{i}=\sum_{j=1, j \neq i}^{k} \mathbf{H}_{j i} \mathbf{Q}_{j} \mathbf{H}_{j i}^{H}+\mathbf{N}_{i}
$$

with $\mathbf{N}_{i}$ being the covariance matrix of the additive noise plus the intercell interference at SR $i$ and being defined as $\mathbf{N}_{i}=\mathbb{E}\left\{\mathbf{n}_{i} \mathbf{n}_{i}^{h}\right\}$. Then, the achievable sum-rate of the $k$ D2D user pairs can be given as

$$
R(\mathbf{Q})=\sum_{i=1}^{k} R_{i}(\mathbf{Q}) .
$$

Since the BS has power to control all D2D user pairs, a maximum tolerable interference power constraint is imposed to protect the signal received by itself. And this coupling interference power constraint can be specifically written as

$$
\sum_{i=1}^{k} \operatorname{Tr}\left(\mathbf{H}_{i 0} \mathbf{Q}_{i} \mathbf{H}_{i 0}^{H}\right) \leq T
$$

where $T>0$ is a predefined maximum interference temperature threshold, and $\mathbf{H}_{i 0} \in \mathbb{C}^{M_{0} \times N_{i}}$ is the complex channel matrix from ST $i$ to the BS.

2.2. Problem Formulation. Then, the SRM problem for optimally coordinated precoding for the $k \mathrm{D} 2 \mathrm{D}$ user pairs can be formulated as

$$
\begin{array}{rl}
\text { (P1): } \max _{\mathbf{Q}} & R(\mathbf{Q}) \\
\text { s.t. } & \operatorname{Tr}\left(\mathbf{Q}_{i}\right) \leq P_{i}, \quad \mathbf{Q}_{i} \geq 0, \forall i \in \mathscr{K}, \\
& \sum_{i=1}^{k} \operatorname{Tr}\left(\mathbf{H}_{i 0} \mathbf{Q}_{i} \mathbf{H}_{i 0}^{H}\right) \leq T,
\end{array}
$$

where $P_{i}>0$ is the maximum transmit power constraint of STi. However, such a SRM problem is notoriously nonconvex and pretty hard to solve. Generally speaking, finding the global optimal strategy profile $\mathbf{Q}^{*}$ for problem (P1) is intractable according to the following lemma.

Lemma 1. Finding the global optimal strategy profile $\mathbf{Q}^{*}$ for problem (P1) is strongly NP-hard.

Proof. If we remove the coupling interference power constraint of problem (P1) and further assume that both the transmitter and the receiver of each D2D user pair employ only one antenna, then problem (P1) can be reduced to a relative "simpler" power control problem, which is stated as follows:

$$
\begin{aligned}
\text { (P0): } \max _{\mathbf{p}} & \sum_{i \in \mathscr{K}} \log \left(1+\frac{p_{i}}{\gamma_{i}+\sum_{j \neq i} \alpha_{j i} p_{j}}\right) \\
\text { s.t. } & 0 \leq p_{i} \leq P_{i},
\end{aligned}
$$

where $\mathbf{p}=\left\{p_{i}\right\}_{i=1}^{k}$ denotes the power strategy profile of all D2D user pairs, $h_{j i}$ is the complex channel gain from ST $j$ to $\mathrm{SR} i, \forall j, i \in \mathscr{K}$, and the parameters $\gamma_{i}$ and $\alpha_{j i}$ are defined as

$$
\begin{gathered}
\gamma_{i}=\frac{\sigma_{i}^{2}}{\left\|h_{i i}\right\|^{2}}, \\
\alpha_{j i}=\frac{\left\|h_{j i}\right\|^{2}}{\left\|h_{i i}\right\|^{2}},
\end{gathered}
$$

with $\sigma_{i}^{2}$ being the noise power level at SRi.

It is already known that the power control problem (P0) is strongly NP-hard according to [11], and the proof is based on a polynomial time transformation from the maximum 
independent set problem [13] (which is known to be NPcomplete). Thus, the SRM problem (P1), as a general case of problem (P0), is also strongly NP-hard.

It is worth noting Lemma 1 roots in a well-known NPhardness result on the power control and precoder design problem for the interfering networks.

In the next section, we will reformulate problem (P1) as a DC-type programming problem, which can be iteratively solved by employing a SCA method.

\section{Centralized Precoding Algorithm}

In this section, a centralized iterative precoding algorithm is developed based on the SCA method to solve problem (P1), and a proximal-point-based regularization approach is also pursued to ensure the convergence.

3.1. Algorithm Design. In order to facilitate the derivations in the following, we reformulate the achievable sum-rate $R(\mathbf{Q})$ of the $k$ D2D user pairs as follows:

$$
R(\mathbf{Q})=\phi(\mathbf{Q})-\varphi(\mathbf{Q}),
$$

where the function $\phi(\mathbf{Q})$ is defined as

$$
\phi(\mathbf{Q})=\sum_{i=1}^{k} \log \left|\mathbf{H}_{i i} \mathbf{Q}_{i} \mathbf{H}_{i i}^{H}+\mathbf{Z}_{i}\right|,
$$

and the function $\varphi(\mathbf{Q})$ is given by

$$
\varphi(\mathbf{Q})=\sum_{i=1}^{k} \log \left|\mathbf{Z}_{i}\right|
$$

Obviously, both $\phi(\mathbf{Q})$ and $\varphi(\mathbf{Q})$ are concave over $\mathbf{Q}$. Hence, the objective function $R(\mathbf{Q})$ is naturally of $\mathrm{DC}$ form, and thus problem (P1) constitutes a DC-type programming problem. Such a problem can be iteratively solved by locally linearizing $\varphi(\mathbf{Q})$ to an affine function, which is detailed as follows.

According to [23], the first-order differential of the function $\varphi(\mathbf{Q})$ can be calculated as

$$
\begin{aligned}
d \varphi(\mathbf{Q}) & =\sum_{i=1}^{k}\left[\sum_{j \neq i}^{k} \operatorname{Tr}\left(\mathbf{H}_{j i}^{H} \mathbf{Z}_{i}^{-1} \mathbf{H}_{j i} d \mathbf{Q}_{j}\right)\right] \\
& =\sum_{j=1}^{k} \operatorname{Tr}\left[\sum_{i \neq j}^{k}\left(\mathbf{H}_{j i}^{H} \mathbf{Z}_{i}^{-1} \mathbf{H}_{j i}\right) d \mathbf{Q}_{j}\right] \\
& =\sum_{j=1}^{k} \operatorname{Tr}\left(\mathbf{D}_{j} d \mathbf{Q}_{j}\right),
\end{aligned}
$$

where $\mathbf{D}_{j}$ is computed as

$$
\mathbf{D}_{j}=\sum_{i=1, i \neq j}^{k} \mathbf{H}_{j i}^{H}\left(\mathbf{Z}_{i}\right)^{-1} \mathbf{H}_{j i} \text {. }
$$

Therefore, around a given strategy profile $\mathbf{Q}^{v}$, the concave function $\varphi(\mathbf{Q})$ can be locally linearized to

$$
\varphi(\mathbf{Q}) \cong \varphi\left(\mathbf{Q}^{v}\right)+\sum_{j=1}^{k} \operatorname{Tr}\left[\mathbf{D}_{j}^{v}\left(\mathbf{Q}_{j}-\mathbf{Q}_{j}^{v}\right)\right]
$$

where $\mathbf{D}_{j}^{v}$ is computed as

$$
\mathbf{D}_{j}^{v}=\sum_{i=1, i \neq j}^{k} \mathbf{H}_{j i}^{H}\left(\mathbf{Z}_{i}^{v}\right)^{-1} \mathbf{H}_{j i}
$$

with $\mathbf{Z}_{i}^{v}$ being calculated as

$$
\mathbf{Z}_{i}^{v}=\sum_{j=1, j \neq i}^{k} \mathbf{H}_{j i} \mathbf{Q}_{j}^{v} \mathbf{H}_{j i}^{H}+\mathbf{N}_{i}
$$

from the given strategy profile $\mathbf{Q}^{v}$.

Then, for a given strategy profile $\mathbf{Q}^{v}$, the local optimal strategy profile $\mathbf{Q}^{*}$ can be obtained by solving the following convex optimization problem [24]:

$$
\begin{aligned}
&(\mathrm{P} 2): \max _{\mathbf{Q}} \quad \phi(\mathbf{Q})-\sum_{i=1}^{k} \operatorname{Tr}\left(\mathbf{D}_{i}^{v} \mathbf{Q}_{i}\right) \\
& \text { s.t. } \quad \operatorname{Tr}\left(\mathbf{Q}_{i}\right) \leq P_{i}, \quad \mathbf{Q}_{i} \geq 0, \quad \forall i \in \mathscr{K}, \\
& \sum_{i=1}^{k} \operatorname{Tr}\left(\mathbf{H}_{i 0} \mathbf{Q}_{i} \mathbf{H}_{i 0}^{H}\right) \leq T,
\end{aligned}
$$

where the constant terms in the objective function have been discarded. Note that the convexity of problem (P2) is built on the assumption that all channel matrices have full row ranks.

Therefore, with the given strategy profile $\mathbf{Q}^{v}$ obtained in the $n$th iteration, problem (P2) can be solved by a standard convex programming algorithm in the $(n+1)$ th iteration. Thus, the nonconvex problem (P1) can be iteratively solved through successive convex programming of problem (P2), for example, with CVX solver [22]. Then, relying on solving series of convexified problems, that is, problem (P2), a centralized iterative precoding algorithm for solving problem (P1) can be developed, which is formally summarized as Algorithm 1.

3.2. Convergence Analysis. Since problem (P1) is generally nonconvex, the convergence behavior of Algorithm 1 with local convex approximation has to be analytically established in the following theorem.

Theorem 2. Suppose that problem (P2) is strictly convex over $\mathbf{Q}$; then from any feasible point $\mathbf{Q}^{v}$, Algorithm 1 will always converge to maxima to problem (P1) by iteratively solving the problem (P2).

Proof. Since the function $\varphi(\mathbf{Q})$ is concave over $\mathbf{Q}$, its firstorder Taylor expansion around any feasible point is always 
(1) initially set $\mathbf{H}_{i j}, \mathbf{H}_{i 0}, P_{i}, \forall i, j \in \mathscr{K}, T, \mathbf{Q}^{v}$, and $v=0$.

(2) repeat

(3) compute $\mathbf{Z}_{i}^{v}, i \in \mathscr{K}$ with $\mathbf{Q}^{v}$, and compute $\mathbf{D}_{j}^{v}, j \in \mathscr{K}$ with $\mathbf{Z}_{i}^{v}$.

(4) compute $\mathbf{Q}^{*}$ by solving problem (P2) with CVX solver [22].

(5) update $v=v+1$, and update $\mathbf{Q}^{v}$ with $\mathbf{Q}^{*}$.

(6) compute $R^{v}=R\left(\mathbf{Q}^{v}\right)$ according to (4)

(7) until the termination criteria is satisfied.

(8) return $R^{v}$ and $\mathbf{Q}^{v}$.

Algorithm 1: Centralized precoding algorithm for solving problem (P1).

larger than itself. This is to say that we can always have the following inequality:

$$
\varphi(\mathbf{Q}) \leq \varphi\left(\mathbf{Q}^{v}\right)+\sum_{j=1}^{k} \operatorname{Tr}\left[\mathbf{D}_{j}^{v}\left(\mathbf{Q}_{j}-\mathbf{Q}_{j}^{v}\right)\right]
$$

for any feasible given strategy profile $\mathbf{Q}^{v}$. Then, it can be concluded that the following inequality

$$
R(\mathbf{Q}) \geq \phi(\mathbf{Q})-\varphi\left(\mathbf{Q}^{v}\right)-\sum_{j=1}^{k}\left\{\operatorname{Tr}\left[\mathbf{D}_{j}^{v}\left(\mathbf{Q}_{j}-\mathbf{Q}_{j}^{v}\right)\right]\right\}
$$

for any given strategy profile $\mathbf{Q}^{v}$ always holds. Thus, it can be concluded that the series values $R^{v}$, defined in Algorithm 1, obtained by employing Algorithm 1 are always upper bounded by

$$
R^{v} \leq R\left(\mathbf{Q}^{*}\right)
$$

where $\mathbf{Q}^{*}$ is the maxima of $R(\mathbf{Q})$ under the given transmit power constraints and the given interference power constraint.

On the other hand, since problem (P2) is supposed to be strictly convex, the series values $R^{v}$ cannot be decreased during the iterations. Hence, the convergence of the proposed iterative precoding algorithm, that is, Algorithm 1, is thus guaranteed, because a monotonically nondecreasing sequence that is upper bounded always converges.

At the same time, it can also conclude that there must exist a limit point of the strategy profile sequence $\mathbf{Q}^{v}$ generated by employing Algorithm 1, and the limit point must constitute a stationary point of problem (P2). Since a stationary point of problem (P2) is also maxima of problem (P1), thus the limit point generated by employing Algorithm 1 must constitute a maxima solution to problem (P1).

Therefore, it can be concluded that Algorithm 1 will always converge to a maxima solution to problem (P1) under the condition that problem (P2) is strictly convex over $\mathbf{Q}$.

According to Theorem 2, it can be concluded that the convergence of Algorithm 1 is established on the strict convexity of problem (P2). However, such a condition is not always satisfied, especially when the MIMO channels are spatially correlated. Therefore, a proximal-point-based regularization approach is pursued in the next subsection to ensure the convergence without requiring any special restrictions on the antenna configurations and also the channel ranks.
3.3. Regularized Approximation. The basic idea of the proximal-point-based regularization consists in penalizing the objective function of problem (P2) using a quadratic regularization term, and thus we can have the following convex optimization problem:

$$
\begin{aligned}
&(\mathrm{P} 3): \max _{\mathbf{Q}} \quad \phi(\mathbf{Q})-\sum_{i=1}^{k}\left[\operatorname{Tr}\left(\mathbf{D}_{i}^{v} \mathbf{Q}_{i}\right)+\tau\left\|\mathbf{Q}_{i}-\mathbf{Q}_{i}^{v}\right\|_{F}^{2}\right] \\
& \text { s.t. } \quad \operatorname{Tr}\left(\mathbf{Q}_{i}\right) \leq P_{i}, \quad \mathbf{Q}_{i} \geq 0, \forall i \in \mathscr{K}, \\
& \sum_{i=1}^{k} \operatorname{Tr}\left(\mathbf{H}_{i 0} \mathbf{Q}_{i} \mathbf{H}_{i 0}^{H}\right) \leq T
\end{aligned}
$$

where $\tau>0$ is a small value to force $\mathbf{Q}$ to stay "close" to $\mathbf{Q}^{v}$. According to the convexity of the Frobenius norm, the strict convexity of problem (P3) can be guaranteed with $\tau>$ 0 without requiring any special restrictions on the antenna configurations and the channel ranks.

The iterative precoding scheme with proximal-pointbased regularization can also be given as Algorithm 1, just with problem $(\mathrm{P} 3)$ replacing problem $(\mathrm{P} 2)$. In order to avoid the ambiguity, the proximal-point-based iterative precoding algorithm will be hereafter referred to as Algorithm 1(P). The convergence behavior of the resultant precoding scheme is analyzed in the following theorem.

Theorem 3. Without requiring any special restrictions on the antenna configurations and the channel ranks, Algorithm 1(P) will always converge to a maxima solution to problem (P1) by iteratively solving problem (P3).

Proof. Because $\tau\left\|\mathbf{Q}_{i}-\mathbf{Q}_{i}^{v}\right\|_{F}^{2}, \tau>0$ is strictly convex over $\mathbf{Q}_{i}$, the strict convexity of problem (P3) is thus established for any possible antenna configurations and the channel ranks. Then, according to the analysis of Algorithm 1 in Theorem 2, it can be concluded that the convergence of Algorithm $1(\mathrm{P})$ is also guaranteed.

Suppose $\mathbf{Q}^{v}$ is the strategy profile sequence generated by employing Algorithm 1(P); according to the analysis of Theorem 2, it can be concluded that the limit point of the sequence $\mathbf{Q}^{v}$ constitutes a stationary point of problem (P3). At the same time, we have

$$
\lim _{n \rightarrow \infty}\left\|\mathbf{Q}^{v+1}-\mathbf{Q}^{v}\right\|_{F}^{2}=0,
$$


which implies that the limit point of $\mathbf{Q}^{v}$ also constitutes a stationary point of problem (P2), because regularization term $\tau\left\|\mathbf{Q}_{i}-\mathbf{Q}_{i}^{v}\right\|_{F}^{2}, \forall i$, is equal to zero at the limit point. Thus, the limit point of $\mathbf{Q}^{v}$ must constitute a feasible solution to problem (P1) as employing Algorithm 1.

Therefore, it can be concluded that Algorithm 1(P) always converges to a maxima solution to problem (P1).

According to Theorem 3, it can be concluded that Algorithm $1(\mathrm{P})$ always converges to a feasible solution to problem (P1) for any possible antenna configurations and the channel ranks. However, the price to pay is that a possibly slower convergence rate is common to the proximal-point-based approximation algorithm.

Thus, the coordinated precoding problem for the D2D user pairs has been solved in a centralized iterative precoding fashion. However, as indicated by many works, high signaling communication cost is always associated with the centralized algorithm. To alleviate the signaling communication cost, a distributed precoding algorithm is often more desirable in practice. Fortunately, such a centralized precoding algorithm can also lend itself to a distributed implementation, which will be introduced in the next section.

\section{Distributed Precoding Algorithm}

In this section, with the aid of a price-based interference management mechanism to overcome the coupling interference power constraint, we reformulate the coordinated precoding problem for the proposed system as a Stackelberg game, where the $\mathrm{BS}$ is assumed as a leader and the $\mathrm{D} 2 \mathrm{D}$ user pairs are modeled as multiple followers. Then, a distributed precoding algorithm is further developed based on the concept of SE.

4.1. Stackelberg Game Formulation. Let us restate the coupling interference power constraint imposed by the BS as follows:

$$
\sum_{i=1}^{k} \operatorname{Tr}\left(\mathbf{H}_{i 0} \mathbf{Q}_{i} \mathbf{H}_{i 0}^{H}\right) \leq T
$$

As mentioned before, the maximum total interference power threshold that the BS can tolerate is $T$; that is, the aggregate interference power from all D2D user pairs cannot be larger than $T$. Then, within the maximum interference power constraint $T$, the BS can also make some profit by selling the interference power quota to each D2D user pair, while protecting itself through pricing the interference power from each D2D user pair. Thus, the coupling interference power constraint can be made separable, which will pave the way for a distributed implementation.

Furthermore, the Stackelberg game model is thus applied for this scenario, which consists of a leader and several followers competing with each other on certain resources. In this work, we assume the BS as the leader and the D2D user pairs as the followers. The price-based interference management mechanism can be detailed as follows: the leader first imposes a price per unit of the interference power for all followers; then, the followers can update their transmit strategies to maximize their own utilities based on the assigned interference power price; and in turn, the leader can update the interference power price according to some rule to maximize his own utility. With properly designed utility functions for the leader and also for each follower, a distributed precoding algorithm can thus be developed in the framework of the Stackelberg game theory.

With the Stackelberg game model, it is easy to observe that the leader's objective is to maximize its own utility obtained by selling the interference power quota to all followers. Formally, the utility function of the leader can be given as follows:

$$
U_{p}(\mu, \mathbf{Q})=\sum_{i=1}^{k} \mu \operatorname{Tr}\left(\mathbf{H}_{i 0} \mathbf{Q}_{i} \mathbf{H}_{i 0}^{H}\right)
$$

where $\mu$ is the uniform interference power price imposed by the $\mathrm{BS}$, which means that the interference power price is equal for all followers. Under the imposed interference power price imposed by the BS, the amount of interference power quota that each D2D user pair wants to buy depends on its own will to maximize its own utility.

Because the maximum aggregate interference power that the BS can tolerate is limited, the BS needs to find the optimal interference price $\mu$ in order to maximize its utility. Given that the followers' transmit strategies remain unchanged, the leader's optimal interference power price can be determined by solving the following convex optimization problem:

$$
\begin{aligned}
\text { (P4.1): } \max _{\mu>0} & U_{p}(\mu, \mathbf{Q}) \\
\text { s.t. } & \sum_{i=1}^{k} \operatorname{Tr}\left(\mathbf{H}_{i 0} \mathbf{Q}_{i} \mathbf{H}_{i 0}^{H}\right) \leq T .
\end{aligned}
$$

Interestingly, it can be observed that the objective function of problem (P4.1) is monotonically increased with $\mu$ under the condition that the followers' transmit strategies remain unchanged. Therefore, the objective function is maximized if and only if the interference power constraint of problem (P4.1) is satisfied with equality. Such a nice property of problem (P4.1) can be further explored for the following algorithm design.

At the D2D user pairs' side, the utility function of the $i$ th $\mathrm{D} 2 \mathrm{D}$ user pair can be defined as

$$
\begin{aligned}
U_{i}\left(\mathbf{Q}_{i}, \mathbf{Q}_{-i}, \mu\right)= & \log \left|\mathbf{H}_{i i} \mathbf{Q}_{i} \mathbf{H}_{i i}^{H}+\mathbf{Z}_{i}^{v}\right| \\
& -\left[\operatorname{Tr}\left(\mathbf{D}_{i}^{v} \mathbf{Q}_{i}\right)+\tau\left\|\mathbf{Q}_{i}-\mathbf{Q}_{i}^{v}\right\|_{F}^{2}\right] \\
& -\mu \operatorname{Tr}\left(\mathbf{H}_{i 0} \mathbf{Q}_{i} \mathbf{H}_{i 0}^{H}\right),
\end{aligned}
$$

where $\mathbf{D}_{i}^{v}$ and $\mathbf{Z}_{i}^{v}$ have the same meaning as defined in (15) and (16) and $\mathbf{Q}_{-i}$ is the strategy profile of all D2D user pairs other than the $i$ th user pair, which is defined as $\mathbf{Q}_{-i}=\left\{\mathbf{Q}_{j}\right\}_{j \neq i}^{k}$. Note that each D2D user pair's utility function defined in (26) comes from problem (P3), where the constant term is also discarded. 


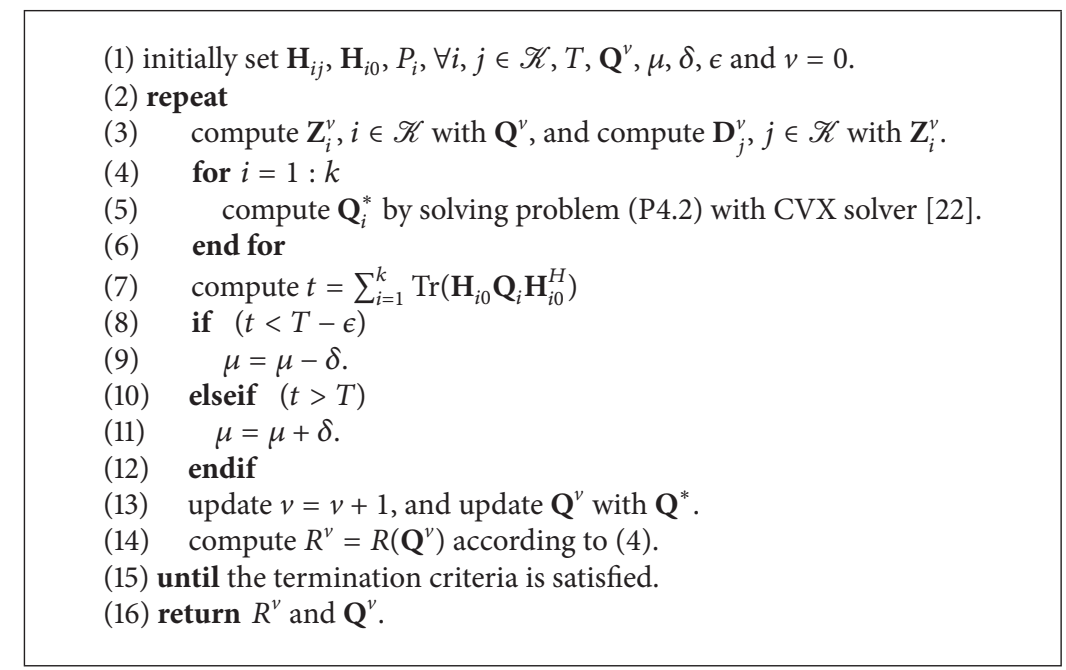

Algorithm 2: Distributed precoding algorithm for solving problem (P1).

Then, under a given interference power price $\mu$ imposed by the BS, the best-response problem for D2D user pair $i, \forall i \in \mathscr{K}$, can be formally formulated as

$$
\begin{aligned}
\text { (P4.2): } \max _{\mathbf{Q}_{i}} & U_{i}\left(\mathbf{Q}_{i}, \mathbf{Q}_{-i}, \mu\right) \\
\text { s.t. } & \operatorname{Tr}\left(\mathbf{Q}_{i}\right) \leq P_{i}, \quad \mathbf{Q}_{i} \geq 0 .
\end{aligned}
$$

Note that problem (P4.2) is convex over $\mathbf{Q}_{i}$ under the condition that $\mathbf{Q}_{-i}$ and $\mu$ remain unchanged.

Therefore, problems (P4.1) and (P4.2) together form a Stackelberg game. The objective of this game is to find a SE solution from which neither the leader nor the followers have incentives to deviate. Formally, the SE for the proposed Stackelberg game can be defined as follows.

Definition of SE. Let $\mu^{*}$ be a solution of problem (P4.1) and let $\mathbf{Q}_{i}^{*}$ be a solution of problem (P4.2) for the $i$ th $\mathrm{D} 2 \mathrm{D}$ user pair. Then, the solution $\left(\mu^{*}, \mathbf{Q}^{*}\right)$ constitutes a SE of the proposed game if the following conditions are satisfied:

$$
\begin{gathered}
U_{p}\left(\mu^{*}, \mathbf{Q}^{*}\right) \geq U_{p}\left(\mu, \mathbf{Q}^{*}\right) \\
U_{i}\left(\mathbf{Q}_{i}^{*}, \mathbf{Q}_{-i}^{*}, \mu^{*}\right) \geq U_{i}\left(\mathbf{Q}_{i}, \mathbf{Q}_{-i}^{*}, \mu^{*}\right), \quad \forall i .
\end{gathered}
$$

Then, a distributed precoding algorithm can be developed based on the concept of SE solution, which will be investigated in the following subsection.

4.2. Algorithm Design. Generally speaking, the SE solution for a Stackelberg game can be obtained by finding its subgames' perfect Nash equilibrium (NE).

At the followers' side, it is not difficult to see from problem (P4.2) that the followers strictly compete in a noncooperative fashion. Thus, through problem (P4.2), a noncooperative subgame has been formulated, and the NE solution of this subgame can be obtained as an operating point. Since problem (P4.2) is strictly convex for each D2D user pair, the NE solution of this subgame can be obtained by convex programming over each $\mathrm{D} 2 \mathrm{D}$ user pair.
At the leader's side, since there exists only one player, the best-response problem can be readily obtained by solving problem (P4.1). However, it is not intended for employing the best-response dynamic for solving problem (P4.1) at the BS side. Instead, a better-response dynamic is preferred here for the interference power price updating, where the above-mentioned nice property of problem (P4.1) will be utilized for the determination of the interference power price.

Specifically, the SE solution for the proposed Stackelberg game can be obtained as follows: for a given $\mu$, problem (P4.2) for each follower is solved by a standard convex programming algorithm based on the best-response dynamic; then, with the obtained best-response strategy profile $\mathbf{Q}^{*}$ for all followers, a better $\mu$ can be decided by resorting to the nice property of problem (P4.1); that is, if the total interference power constraints exceed $T$, then a higher price will be imposed; otherwise, a relative lower price will be imposed; then, a new round of competition between the D2D user pairs will be started until a SE solution is reached. Thus, a distributed iterative algorithm can be developed for the SE solution of the proposed Stackelberg game, which is formally summarized as Algorithm 2. Note that the parameter $\epsilon$ employed in Algorithm 2 is a preset precision parameter to ensure a tolerance, while $\delta$ is a step parameter for the interference power price updating.

It can be seen that Algorithm 2 is a totally distributed iterative precoding algorithm. At the BS's side, the BS only needs to measure the total received interference power, and then a decision can be made to update the interference power price. At the D2D user pairs' side, each D2D user pair only needs to know its own channel matrix and the channel matrix from itself to the BS. Thus, the amount of signaling information that needs to be exchanged is greatly reduced as compared with the centralized precoding algorithm.

4.3. Stackelberg Equilibrium Analysis. In this subsection, the property of the SE solution for the proposed game is analyzed. 


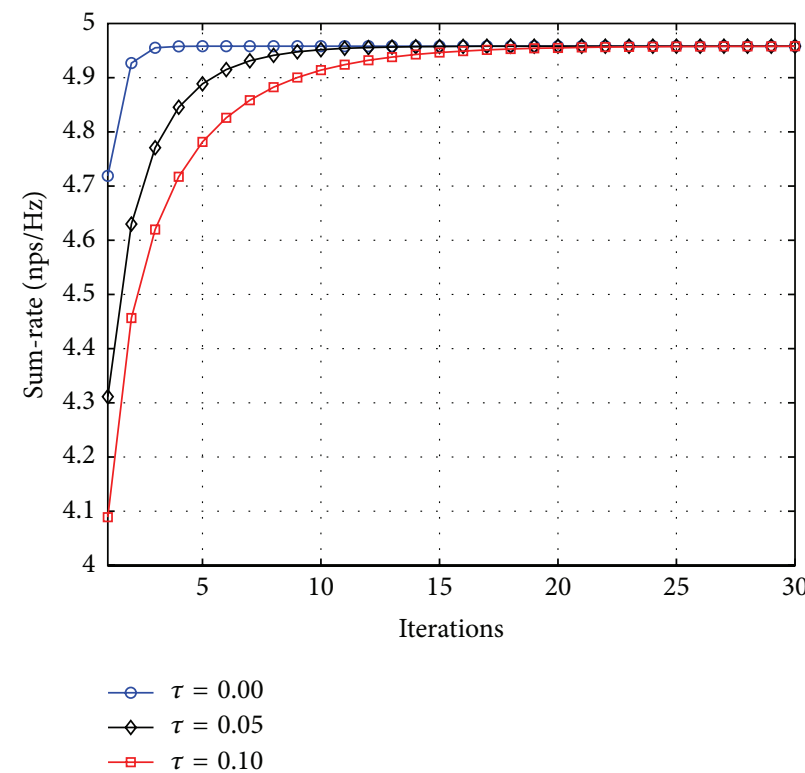

(a) $T=0.5$

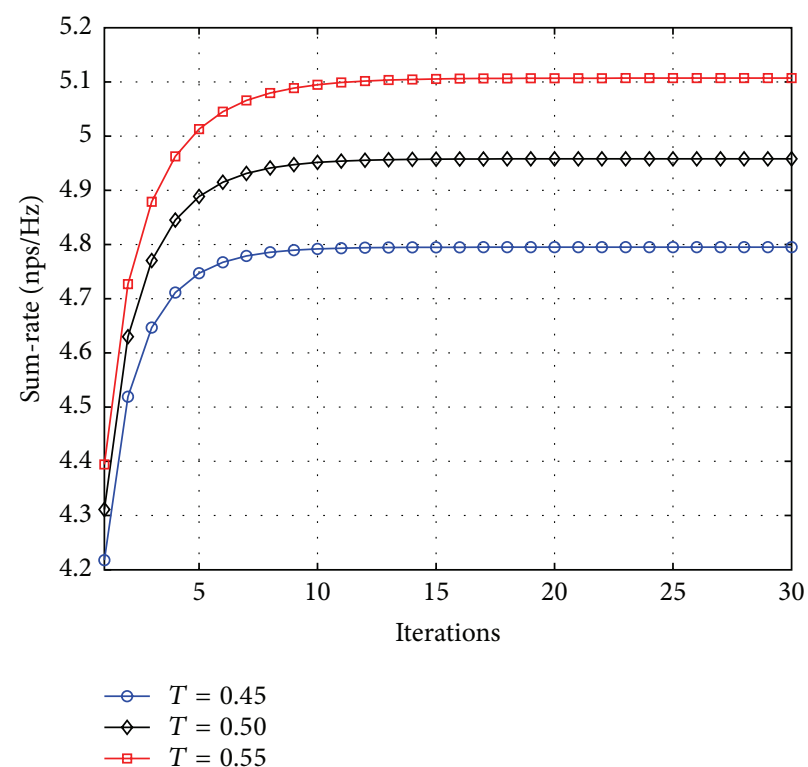

(b) $\tau=0.05$

FIGURE 2: Convergence behavior of Algorithm 1(P).

Theorem 4. With a given interference price $\mu$, subgame (P4.2) constitutes a potential game [25]. Moreover, the optimal solution $\mathbf{Q}^{*}$ of problem (P1) constitutes a Pareto optimal pure strategy NE of subgame (P4.2) under the optimal interference price $\mu^{*}$.

Proof. With a given interference price $\mu$, the total utility of all D2D user pairs can be given as

$$
\begin{aligned}
U(\mathbf{Q}, \mu)= & \sum_{i=1}^{k} U_{i}\left(\mathbf{Q}_{i}, \mathbf{Q}_{-i}, \mu\right) \\
= & \sum_{i=1}^{k} \log \left|\mathbf{H}_{i i} \mathbf{Q}_{i} \mathbf{H}_{i i}^{H}+\mathbf{Z}_{i}^{v}\right| \\
& -\sum_{i=1}^{k}\left[\operatorname{Tr}\left(\mathbf{D}_{i}^{v} \mathbf{Q}_{i}\right)+\tau\left\|\mathbf{Q}_{i}-\mathbf{Q}_{i}^{v}\right\|_{F}^{2}\right] \\
& -\mu \sum_{i=1}^{k} \operatorname{Tr}\left(\mathbf{H}_{i 0} \mathbf{Q}_{i} \mathbf{H}_{i 0}^{H}\right) .
\end{aligned}
$$

Then, $\forall i \in \mathscr{K}$, we have

$$
\begin{aligned}
& U_{i}\left(\mathbf{Q}_{i}, \mathbf{Q}_{-i}\right)-U_{i}\left(\mathbf{Q}_{i}^{\prime}, \mathbf{Q}_{-i}\right) \\
& \quad=U\left(\mathbf{Q}_{i}, \mathbf{Q}_{-i}\right)-U\left(\mathbf{Q}_{i}^{\prime}, \mathbf{Q}_{-i}\right) .
\end{aligned}
$$

This means that subgame (P4.2) is a potential game and $U(\mathbf{Q}, \mu)$ is the potential function. With the optimal interference price $\mu^{*}$, the constraint of problem (P4.1) must be satisfied with equality when the NE of game (P4.2) is reached. That is to say,

$$
\sum_{i=1}^{k} \operatorname{Tr}\left(\mathbf{H}_{i 0} \mathbf{Q}_{i} \mathbf{H}_{i 0}^{H}\right)=T
$$

must be satisfied.

On the other hand, maxima $\mathbf{Q}^{*}$ of problem (P1) is reached; the interference power constraint of it must also be satisfied with equality. This means that we also have formula (31) with $\mathbf{Q}^{*}$. Moreover, when $\mathbf{Q}^{*}$ is reached, the sum-rate of all D2D user pairs reaches maxima. At this point, no players can benefit by unilaterally changing their own strategy, and such a point $\mathbf{Q}^{*}$ constitutes a stationary point of the following optimization problem:

$$
\begin{array}{rl}
\text { (P5): } \max _{\mathbf{Q}} & U\left(\mathbf{Q}, \mu^{*}\right) \\
\text { s.t. } & \operatorname{Tr}\left(\mathbf{Q}_{i}\right) \leq P_{i}, \quad \mathbf{Q}_{i} \geq 0, \forall i \in \mathscr{K},
\end{array}
$$

which means that $\mathbf{Q}^{*}$ constitutes a Pareto optimal pure strategy NE of subgame (P4.2).

Based on the property analyzed in Theorem 4, it can be concluded that Algorithm 2 is sure to converge to SE solution of the proposed game, and the SE solution has a potential to be a Pareto optimal solution of problem (P1).

\section{Simulation Examples}

In this section, the proposed algorithms are investigated via numerical simulations.

During the simulations, it is assumed that there are $k=3$ D2D user pairs, and the number of antennas employed by all 


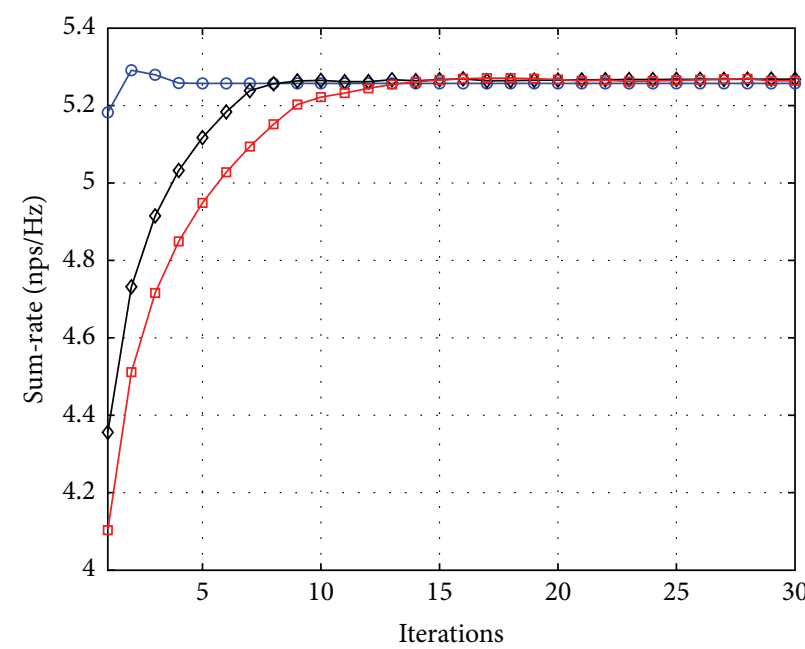

$\multimap \tau=0.00$
$\multimap \tau=0.05$
$\square \tau=0.10$

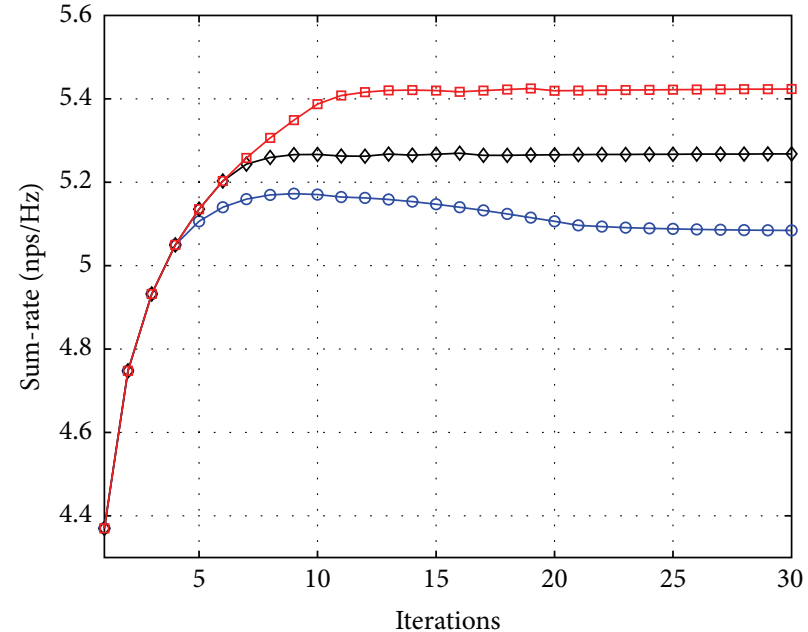

$\multimap T=0.45$
$\multimap T=0.50$
$\multimap \quad T=0.55$

(a) $T=0.5$

(b) $\tau=0.05$

Figure 3: Convergence behavior of Algorithm 2.

STs and SRs is assumed to be equal to $N=2$. The transmit power constraints of all STs are also assumed to be equal to $P=1$. The elements of channel matrix $\mathbf{H}_{i j}$ between $i \in \mathscr{K}$ and $j \in\{0, \mathscr{K}\}$ are all assumed to be i.i.d. ZMCSCG random noise with variances set as $\sigma_{i j}^{2}=0.25, \forall i \neq j$, and $\sigma_{i j}^{2}=1, \forall i=j$. The control parameters of Algorithm 2 are set as $\epsilon=0.01$, while $\mu$ is initialized as 2.7 , and the variable step parameter $\delta$ has a value stated as

$$
\delta= \begin{cases}0.04, & \text { when } n \leq 10 \\ 0.02, & \text { when } n>10\end{cases}
$$

The convergence behavior of Algorithm 1(P) is shown in Figure 2, where (a) is obtained with $T=0.5$ and presented under different $\tau$, while (b) is obtained with $\tau=0.05$ and presented under different $T$ for a comparison. It can be seen from this figure that Algorithm 1(P) always converges to a single point with fixed $T$ and the achievable sum-rate of Algorithm 1(P) is always increased with the relax of the interference power constraint $T$. It can also be seen from this figure that the convergence speed of Algorithm $1(\mathrm{P})$ is slightly slower with the increase of $\tau$.

The convergence behavior of Algorithm 2 is demonstrated in Figure 3, where (a) is also obtained with $T=0.5$ and presented under different $\tau$, while (b) is obtained with $\tau=0.05$ and presented under different $T$ for a comparison. It can also be seen from this figure that the achievable sumrate obtained by Algorithm 2 also increases with the relax of the interference power constraint $T$. Compared with Figure 2, it can be seen that Algorithm 2 and Algorithm 1(P) almost share the same convergence behavior. However, because the problem (P1) is generally nonconvex and more than one maxima point exists, the solutions obtained by Algorithm 2 are not always the same as obtained by Algorithm $1(\mathrm{P})$. As shown by Figure 3, the sum-rate achieved by Algorithm 2 is larger than that achieved by Algorithm 1(P) in Figure 2, both of which are obtained under the same channel environment.

The parameter evolution behavior of Algorithm 2, which is obtained in the simulation of Figure 3(b), is shown in Figure 4 , where the evolution behavior of the total interference power $t$ is presented in (a), while the evolution behavior of the interference price $\mu$ is presented in (b). From Figure 4(a), it can be seen that the total interference power $t$ converges exactly to the predefined $T$ as expected. From Figure 4(b), it can be seen that the cause of the convergence of $t$ is the convergence of the interference price $\mu$.

\section{Conclusion}

In this paper, we have studied the coordinated precoder design problem for D2D communications underlay uplink MIMO cellular networks. First, the problem is formulated as a SRM problem, which can be further reformulated as a DC-type programming problem. Based on the SCA method, a centralized iterative precoding algorithm is developed for a maxima solution. And the proximal-point-based regularization approach is also pursued to ensure the convergence of the proposed algorithm. Second, with the aid of a price-based interference control mechanism to overcome the coupling interference power constraint, we reformulate the coordinated precoding problem as a Stackelberg game. Then, a distributed precoding algorithm is further developed based on the concept SE. Results show that our algorithms can converge fast to a satisfactory solution with guaranteed convergence.

\section{Competing Interests}

The authors declare that they have no competing interests. 


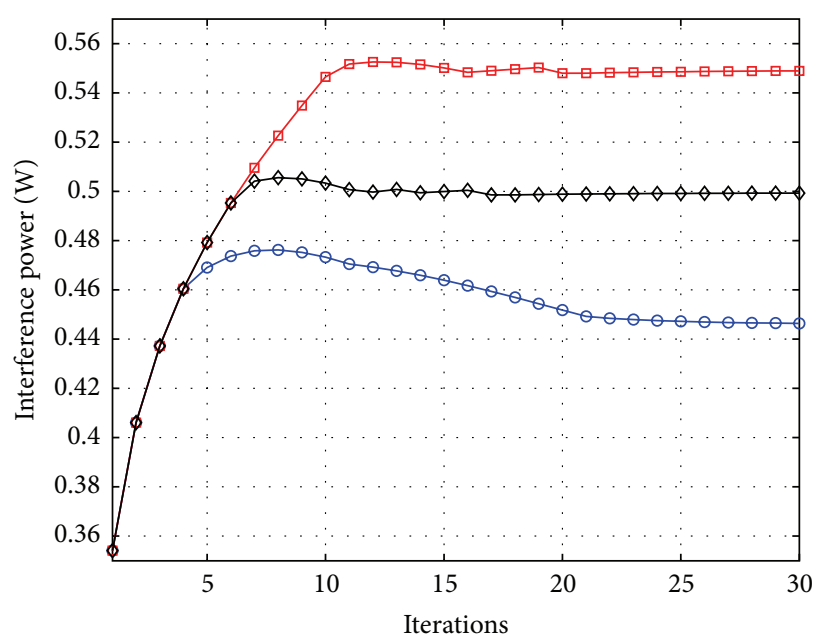

$\multimap T=0.45$
$\longrightarrow T=0.55$
$\multimap T=0.50$

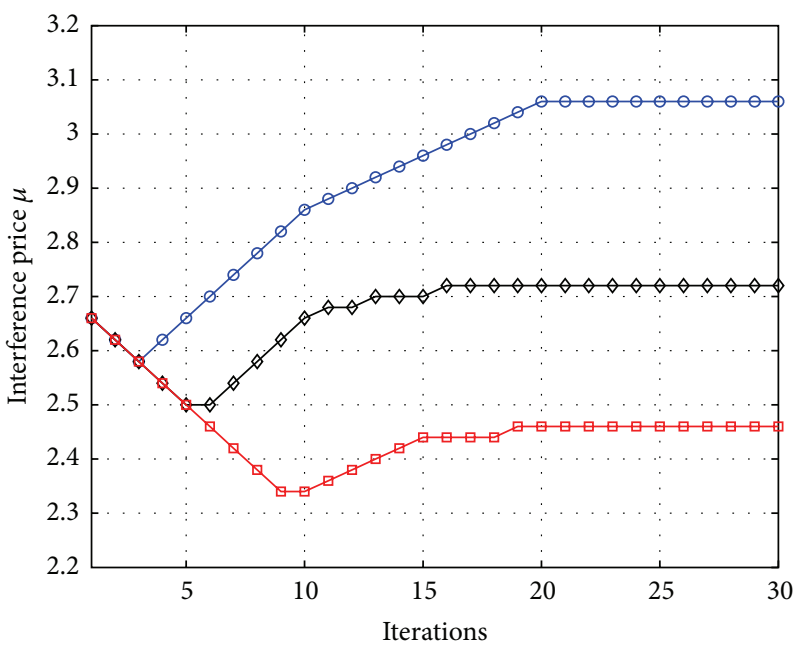

$\multimap T=0.45$
$\multimap T=0.50$
$\square T=0.55$

(b) $\tau=0.05$

(a) $\tau=0.05$

FIgURE 4: Parameter evolution behavior of Algorithm 2.

\section{Acknowledgments}

This work was supported by the Natural Science Foundation of China under Grants 61201218 and 61201241 and by the Project funded by China Postdoctoral Science Foundation under Grants 2013M532207 and 2014T70966.

\section{References}

[1] X. Lin, J. G. Andrews, A. Ghosh, and R. Ratasuk, "An overview of 3GPP device-to-device proximity services," IEEE Communications Magazine, vol. 52, no. 4, pp. 40-48, 2014.

[2] D. Feng, L. Lu, Y. Y. Wu, G. Li, S. Li, and G. Feng, "Device-todevice communications in cellular networks," IEEE Communications Magazine, vol. 52, no. 4, pp. 49-55, 2014.

[3] P. Phunchongharn, E. Hossain, and D. I. Kim, "Resource allocation for device-to-device communications underlaying LTE-advanced networks," IEEE Wireless Communications, vol. 20, no. 4, pp. 91-100, 2013.

[4] D. Zhu, J. Wang, A. L. Swindlehurst, and C. Zhao, "Downlink resource reuse for device-to-device communications underlaying cellular networks," IEEE Signal Processing Letters, vol. 21, no. 5, pp. 531-534, 2014.

[5] C. Xu, L. Song, and Z. Han, Resource Management for Device-toDevice Underlay Communication, SpringerBriefs in Computer Science, Springer, 2013.

[6] J. C. F. Li, M. Lei, and F. Gao, "Device-to-device (D2D) communication in MU-MIMO cellular networks," in Proceedings of the IEEE Global Communications Conference (GLOBECOM '12), pp. 3583-3587, Anaheim, Calif, USA, December 2012.

[7] H. Tang, C. Zhu, and Z. Ding, "Cooperative MIMO precoding for D2D underlay in cellular networks," in Proceedings of the IEEE International Conference on Communications (ICC'13), pp. 5517-5521, Budapest, Hungary, June 2013.

[8] P. Jänis, V. Koivunen, C. B. Ribeiro, K. Doppler, and K. Hugl, "Interference-avoiding MIMO schemes for device-to-device radio underlaying cellular networks," in Proceedings of the IEEE 20th Personal, Indoor and Mobile Radio Communications Symposium (PIMRC '09), pp. 2385-2389, Tokyo, Japan, September 2009.

[9] W. Zhong, Y. Fang, S. Jin, K.-K. Wong, S. Zhong, and Z. Qian, "Joint resource allocation for device-to-device communications underlaying uplink MIMO cellular networks," IEEE Journal on Selected Areas in Communications, vol. 33, no. 1, pp. 41-54, 2015.

[10] X. Chen, B. Proulx, X. Gong, and J. Zhang, "Exploiting social ties for cooperative D2D communications: a mobile social networking case," IEEE/ACM Transactions on Networking, vol. 23, no. 5, pp. 1471-1484, 2015.

[11] Z.-Q. Luo and S. Zhang, "Dynamic spectrum management: complexity and duality," IEEE Journal on Selected Topics in Signal Processing, vol. 2, no. 1, pp. 57-73, 2008.

[12] E. G. Larsson and E. A. Jorswieck, "Competition versus cooperation on the MISO interference channel," IEEE Journal on Selected Areas in Communications, vol. 26, no. 7, pp. 1059-1069, 2008.

[13] Y.-F. Liu, Y.-H. Dai, and Z.-Q. Luo, "Coordinated beamforming for MISO interference channel: complexity analysis and efficient algorithms," IEEE Transactions on Signal Processing, vol. 59, no. 3, pp. 1142-1157, 2011.

[14] W. Zhong and J. Wang, "Energy efficient spectrum sharing strategy selection for cognitive MIMO interference channels," IEEE Transactions on Signal Processing, vol. 61, no. 14, pp. 37053717, 2013.

[15] X. Shang and H. V. Poor, "Noisy-interference sum-rate capacity for vector Gaussian interference channels," IEEE Transactions on Information Theory, vol. 59, no. 1, pp. 132-153, 2013.

[16] Y. Zhang, E. D. Anese, and G. B. Giannakis, "Distributed optimal beamformers for cognitive radios robust to channel uncertainties," IEEE Transactions on Signal Processing, vol. 60, no. 12, pp. 6495-6508, 2012.

[17] A. Alvarado, G. Scutari, and J.-S. Pang, "A new decomposition method for multiuser DC-programming and its applications," 
IEEE Transactions on Signal Processing, vol. 62, no. 11, pp. 29842998, 2014.

[18] J.-S. Pang, G. Scutari, D. P. Palomar, and F. Facchinei, "Design of cognitive radio systems under temperature-interference constraints: a variational inequality approach," IEEE Transactions on Signal Processing, vol. 58, no. 6, pp. 3251-3271, 2010.

[19] J. Wang, G. Scutari, and D. P. Palomar, "Robust MIMO cognitive radio via game theory," IEEE Transactions on Signal Processing, vol. 59, no. 3, pp. 1183-1201, 2011.

[20] X. Kang, R. Zhang, and M. Motani, "Price-based resource allocation for spectrum-sharing femtocell networks: a stackelberg game approach," IEEE Journal on Selected Areas in Communications, vol. 30, no. 3, pp. 538-549, 2012.

[21] J. Wang, M. Peng, S. Jin, and C. Zhao, "A generalized nash equilibrium approach for robust cognitive radio networks via generalized variational inequalities," IEEE Transactions on Wireless Communications, vol. 13, no. 7, pp. 3701-3714, 2014.

[22] CVX Research Inc, CVX: Matlab software for disciplined convex programming, version 2.0, http://cvxr.com/cvx.

[23] X. Zhang, Matrix Analysis and Applications, Tsinghua University Press, Beijing, China, 2004.

[24] S. Boyd and L. Vandenberghe, Convex Optimization, Cambridge University Press, Cambridge, UK, 2004.

[25] D. Monderer and L. S. Shapley, "Potential games," Games and Economic Behavior, vol. 14, no. 1, pp. 124-143, 1996. 

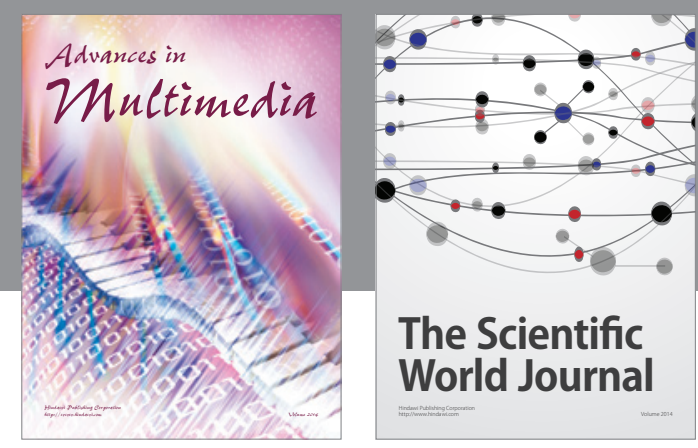

The Scientific World Journal
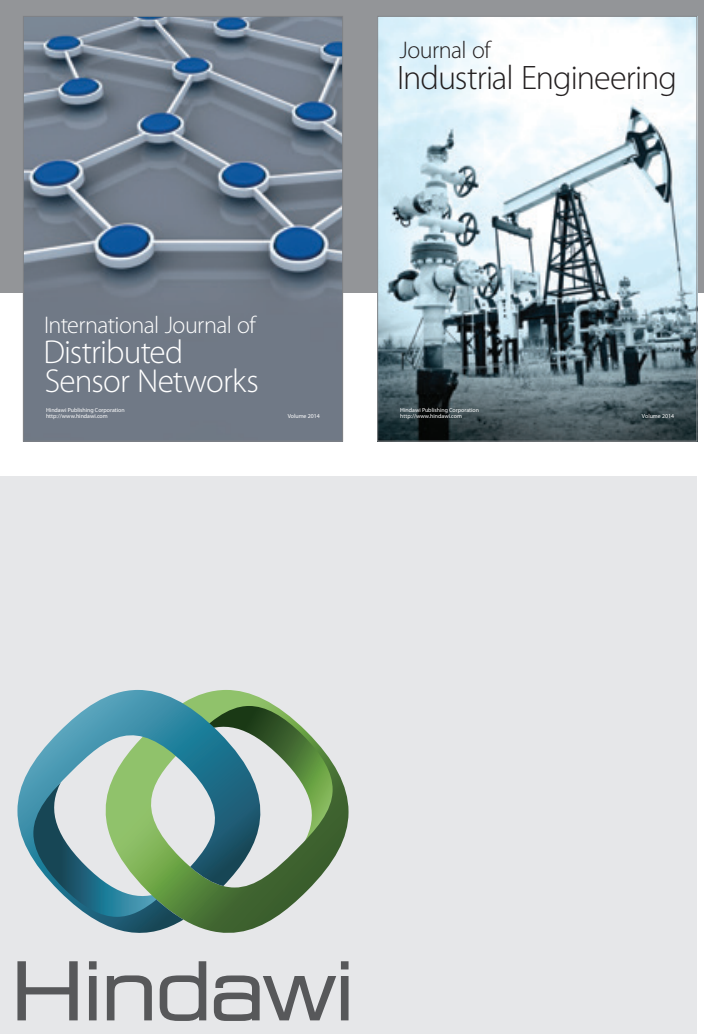

Submit your manuscripts at

http://www.hindawi.com

\section{Computer Networks} and Communications
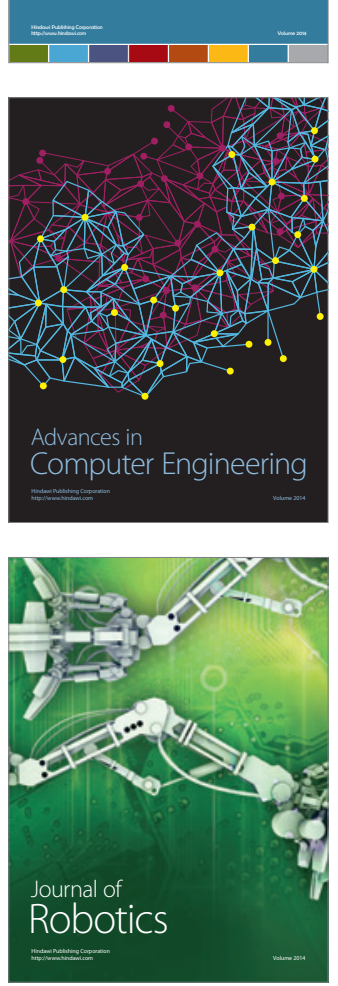
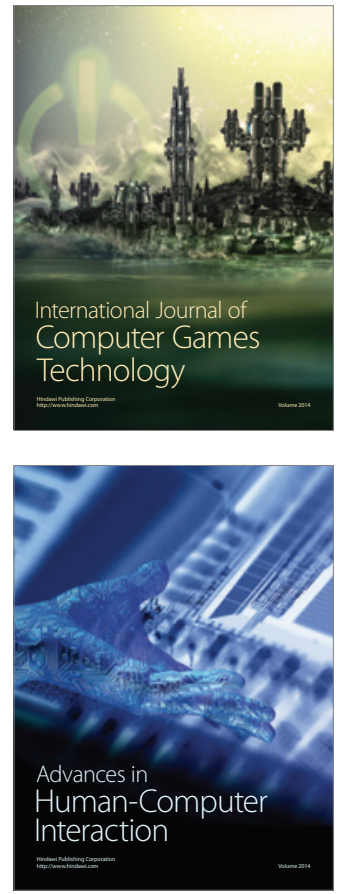
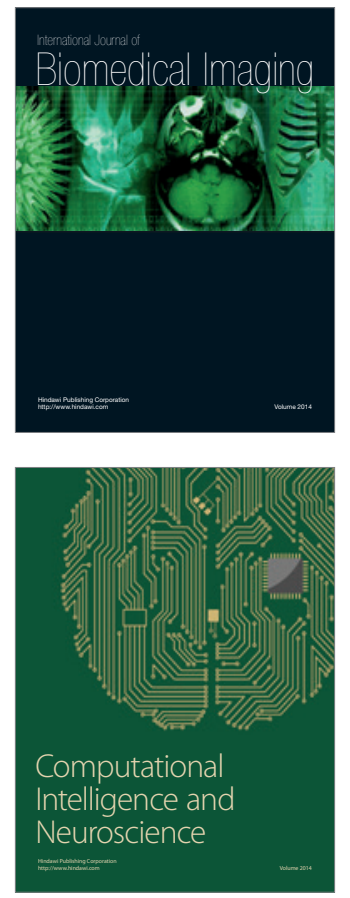
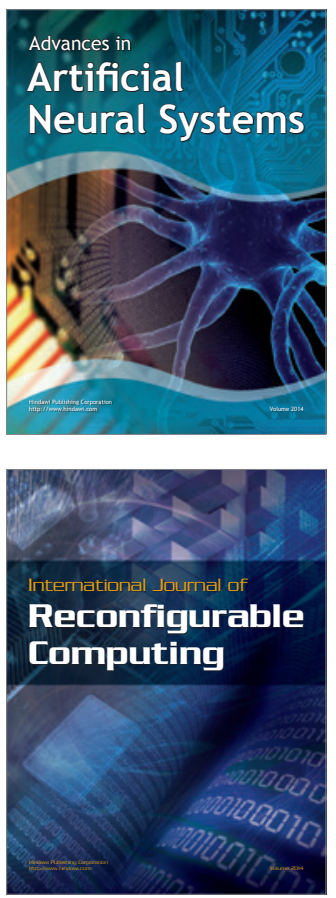
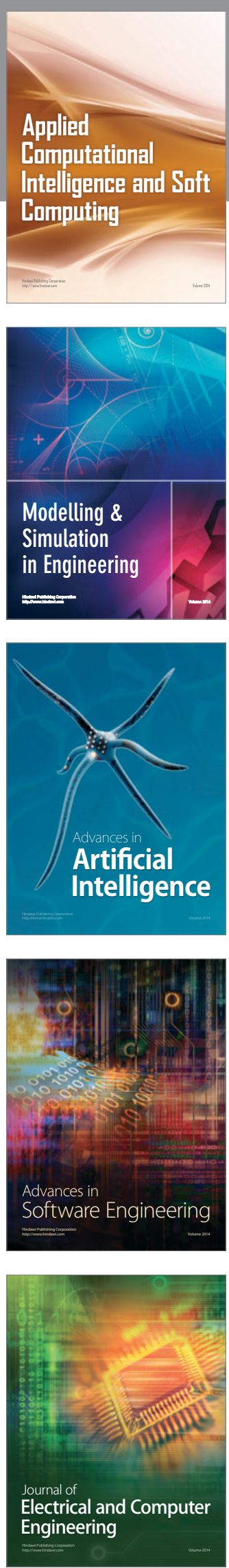